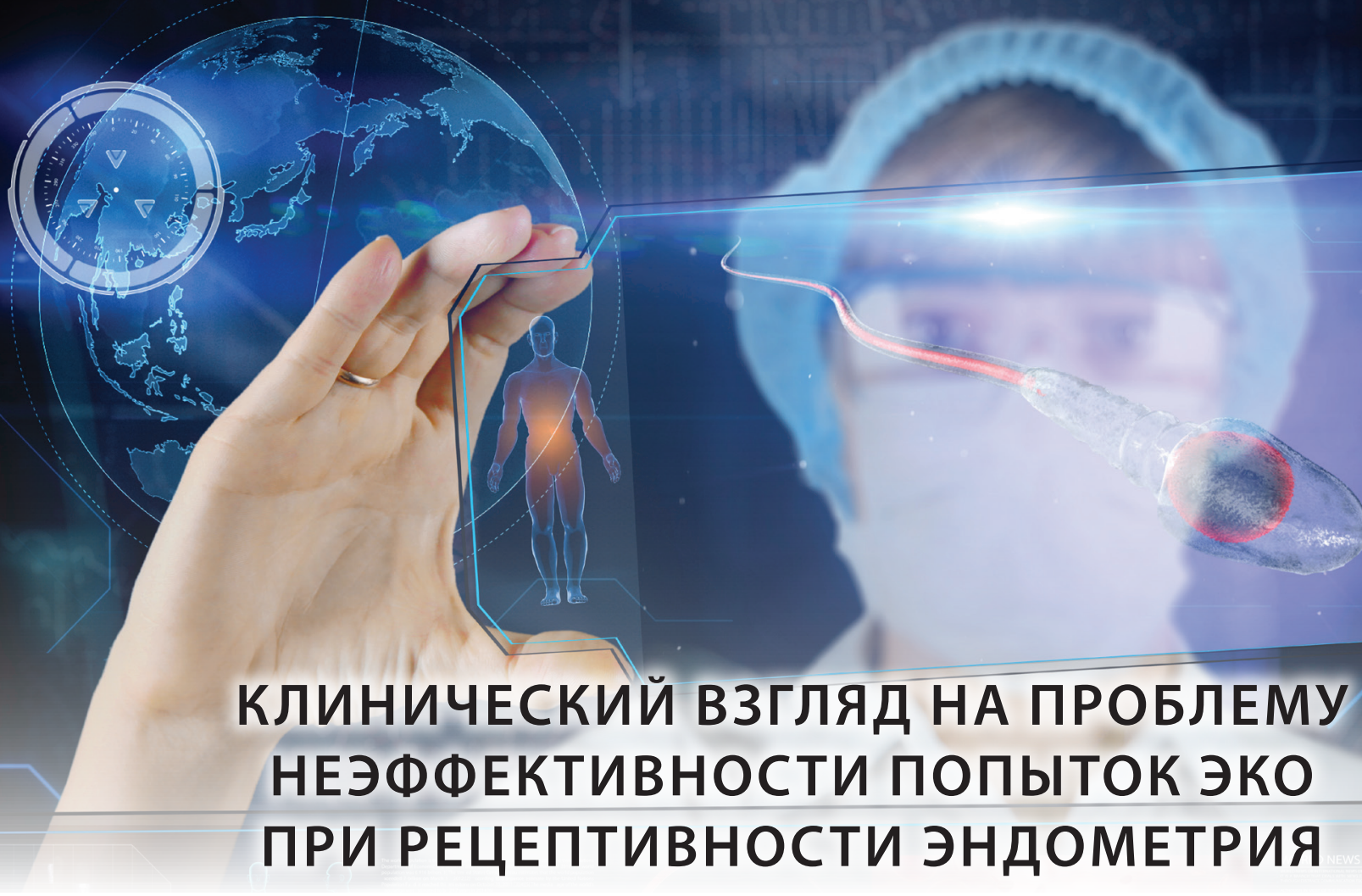

\author{
ОБЗОР ЛИТЕРАТУРЫ
}

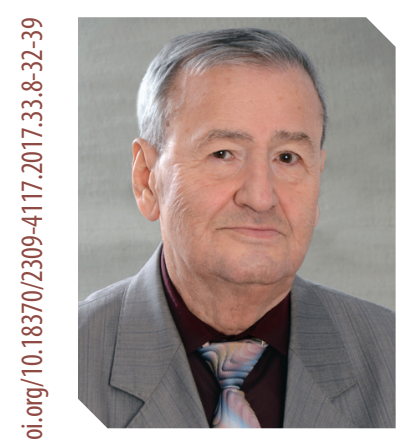

\section{П.Н. ВЕРОПОТВЕЛЯН}

к. мед. н., зав. отделением патологии репродуктивной функции человека Межобластного центра медицинской генетики и пренатальной диагностики, г. Кривой Рог

ORCID: 0000-0002-4367-3630

И.С. ЦЕХМИСТРЕНКО врач акушер-гинеколог Перинатального центра г. Киева ORCID: 0000-0003-0777-2225

Контакты:

Веропотвелян Петр Николаевич ОкУ «Межобластной центр медицинской генетики и пренатальной диагностики», отделение патологии репродуктивной функции человека 50000, Кривой Рог, пл. Освобождения, За тел.: + 380 (564) 923609 e-mail: genetika@ukrpost.ua

\section{ВВЕДЕНИЕ}

Повышение эффективности методов вспомогательных репродуктивных технологий (ВРТ) является актуальной задачей современной репродуктивной медицины. В то же время процесс имплантации эмбриона до сегодняшнего дня остается не до конца изученным этапом, лимитирующим частоту успешных исходов циклов экстракорпорального оплодотворения (ЭКО) [13].

Как сообщают А. Ferraretti и соавторы, наступление клинической беременности и родов живым плодом в циклах ЭКО не превышает $33 \%$ и 24,8\% соответственно [14]. Это связано, во-первых, с низкой вероятностью зачатия и родов у человека, не превышающей у здоровых женщин 19-26 лет при регулярной половой жизни $55 \%$ в расчете на один менструальный цикл (МЦ) [15]. Во-вторых, основной проблемой остается отсутствие имплантации при пересадке эмбриона хорошего качества в полость матки. По расчетам специалистов, на отсутствие имплантации приходится 70\% причин неудач в программах ЭКО.

Повторные неудачи имплантации (recurrent implantation failure, RIF) в программе ЭКО - клиническая ситуация, в которой неоднократно не удается достичь стадии визуализации плодного яйца в полости матки при ультразвуковом исследовании (УЗИ) после переноса эмбриона.

Ведущую роль в процессе имплантации занимает так называемая рецептивность эн- дометрия, т. е. способность последнего обеспечить необходимые этапы имплантации: ориентацию бластоцисты в полости матки относительно места будущей имплантации, адгезию на поверхности эндометрия и инвазию (внедрение) в полость матки. Также исследователями было разработано понятие «окна имплантации», т. е. способности обеспечить её необходимые этапы в определенный период, который составляет несколько дней - примерно с 20-го по 24-й день нормального МЦ, на 6-10-й день после пика лютеинизирующего гормона (ЛГ) $[16,17,18]$.

В любое другое время эндометрий не является рецептивным, т. е. успешная имплантация эмбриона невозможна. Как информирует J. Evans [19], хорошо известно, что уровень сывороточных гормонов в естественном МЦ, который предопределяет развитие эндометрия, значительно меняется в стимулированных циклах программы ЭКО, в связи с чем «окно имплантации» может изменяться во времени или отсутствовать.

Изучение эндометрия в период имплантации началось более 60 лет назад, когда американские ученые четко описали секреторные изменения эндометрия, максимум которых приходится на 7-й день лютеиновой фазы цикла [20]. К сожалению, одно лишь гистологическое исследование не дает представления о рецептивности эндометрия и скорее иллюстрирует овуляцию, произошедшую в данном МЦ. 
Дальнейшие исследования рецептивности эндометрия были связаны с изучением рецепторов эстрогенов и прогестерона в клетках эпителия и стромы эндометрия в период имплантации. Выяснено, что в начале секреторной фазы цикла (на 16-й день идеального МЦ в 28 дней) в клетках эпителия и стромы эндометрия наблюдается большое количество рецепторов эстрогенов и прогестерона. К 20-22 дню цикла, которые считаются временем имплантации бластоцисты, эти рецепторы в клетках эпителия полностью исчезают, в то время как их количество в клетках стромы остается крайне высоким. Следует отметить, что исчезновение рецепторов в клетках эпителия совпадает с наивысшими величинами уровня эстрадиола и прогестерона в крови женщины в течение МЦ. Исследователи считают, что именно резкое снижение уровня рецепторов к эстрогенам и прогестерону в клетках эпителия вызывает появление на поверхности этих клеток белков клеточной адгезии (интегринов), а также увеличивает чувствительность к факторам роста, выделяемым клетками стромы эндометрия [21].

\section{ИНДУКТОРЫ, ВЛИЯЮЩИЕ НА ЭНДОМЕТРИЙ В СТИМУЛИРОВАННЫХ ЦИКЛАХ}

В ряде исследований было показано неблагоприятное воздействие стимуляции суперовуляции на рецептивность эндометрия, где частота имплантации была значительно ниже у доноров ооцитов, чем у соответствующих по возрасту и качеству эмбрионов рецепиентов [22].

B. Shapiro и соавторы, оценивая эндометрий в стимулированных циклах ЭКО в сравнении с натуральным циклом, обнаружили преждевременную секреторную трансформацию и изменение экспрессии маркеров рецептивности в постовуляторной и ранней лютеиновой фазе, что, возможно, приводит к несинхронному взаимодействию между жизнеспособным эмбрионом и рецептивным или субрецептивным эндометрием [23]. Кроме того, по данным исследователей, в лютеиновой фазе стимулированного цикла изменяется профиль экспрессии генов эндометрия [24]. В описанных протоколах с антагонистом гонадотропин-рилизинг гормона (ГнРГ) снижается экспрессия генов НОХА 10 в строме эндометрия.

Ряд ученых не обнаружили различий в экспрессии различных маркеров рецептивности эндометрия при сравнении натуральных и стимулированных циклов овуляции $[25,26]$. Кроме того, не выявлены доказательства негативного влияния овариальной стимуляции на рецептивность эндометрия.

F. Ubaldi, E. Kolibianakic и соавторы изучали в протоколах эффект стимуляции яичников на созревание эндометрия в день получения ооцитов в циклах без отмены переноса как с агонистами ГнРГ (а-ГнРГ), так и антагонистами ГнРГ (антГнРГ) $[27,28]$.

B. Tarlatzis и соавторы предполагали, что применение ант-ГнРГ может оказывать более негативное влияние на эндометрий по сравнению с а-ГнРГ [29]. Но в то же время другие исследователи показали, что эндометрий в протоколе с ант-ГнРГ больше соответствует натуральному циклу, чем в протоколе а-ГнРГ [30]. Так, А. Tavaniotou и коллеги проиллюстрировали, что применение а-ГнРГ в «длинном» протоколе приводит к глубокой десенситизации гипофиза
[16]. В проведенных исследованиях секреции гонадотропинов гипофизом после введения а-ГнРГ показано, что эти нарушения сохраняются по крайней мере на протяжении 14 дней после прекращения введения препаратов, то есть в течение всей лютеиновой фазы. Изменения сохраняются даже при непродолжительном введении а-ГнРГ в фолликулярной фазе стимулированного цикла.

Вышеупомянутые авторы предполагают, что в отличие от а-ГнРГ, применение ант-ГнРГ не может повлиять на секрецию ЛГ в лютеиновой фазе стимулированного цикла, так как гипофиз сохраняет способность реагировать на эндогенный ГнРГ [16]. Тем не менее, в ряде исследований уровень ЛГ в лютеиновую фазу при применении ант-ГнРГ был низким. Значит, применение как а-ГнРГ, так и ант-ГнРГ приводит к снижению секреции ЛГ в лютеиновой фазе стимулированного цикла и, следовательно, к нарушению функции желтого тела и низким уровням прогестерона. Кроме того, нельзя исключить прямое влияние аналогов ГнРГ на эндометрий. Так как клетки эндометрия экспрессируют $\mu$-РНК рецепторов ГнРГ, лечение ант-ГнРГ может негативно влиять на рецептивность эндометрия. Далее исследователи сообщают, что развитие и рост нескольких фолликулов в результате гормональной стимуляции яичников увеличивает количество полученных эмбрионов, доступных для переноса. В этой же работе отмечается, что стимуляция яичников вызывает закономерное увеличение сывороточного уровня эстрадиола (E2) и прогестерона, что по механизму обратной связи приводит к нарушению секреции ЛГ гипофизом и к снижению его уровня в сыворотке крови [16]. Кроме того, известно, что существенные изменения сывороточного уровня стероидных гормонов могут влиять на экспрессию рецепторов в эндометрии, нарушая тем самым его рецептивность.

По данным ряда публикаций, в день назначения препарата хорионического гонадотропина человека (ХГч) для финального созревания ооцитов в циклах стимуляции встречается умеренное повышение уровня сывороточного прогестерона [31, 32]. C. Venetis и соавторы информируют, что феномен, получивший название «преждевременная лютеинизация» (ПЛ), является одной из причин низкой эффективности программ ЭКО и, по разным данным, осложняет от 12,4 до 52,3\% циклов ВРТ с использованием аналогов ГнРГ [33].

В то же время механизмы влияния на рецептивность эндометрия повышенных концентраций прогестерона в день введения триггера остаются невыясненными. Работа исследователей [34] иллюстрирует, что продолжительное повышение концентрации прогестерона приводит к преждевременному созреванию эндометрия и, как следствие, к раннему закрытию «окна имплантации» и снижению частоты наступления беременности. При этом авторы предполагают, что решающее значение для адекватного функционирования «окна имплантации» имеет продолжительность воздействия прогестерона, а не его абсолютный уровень [18, 34]. Кроме того, результаты исследований в программе донации ооцитов показали, что сывороточный уровень прогестерона доноров в конце стимуляции не влиял на частоту наступления беременности реципиентов, что дает право предположить неблагоприятное воздействие ПЛ на эндометрий [32]. 


\section{пИноподИи}

Представленный интерес [34] к проблеме рецептивности эндометрия остается высоким, в связи с чем был предложен ряд новых маркеров «окна имплантации». Одним из них было появление на эндометрии в середине лютеиновой фазы пиноподий. Пиноподии, выросты на поверхности эндометрия (около 5 нм в диаметре), были обнаружены в период, совпадающий с «окном имплантации», с помощью электронной микроскопии у ряда млекопитающих, в частности, крысы и человека. Физиологическое значение развития пиноподий до конца не выяснено [35], однако есть ряд доказательств, что начальные этапы адгезии (прикрепления) бластоцисты к рецептивному эндометрию происходят именно на их поверхности (рис. 1).

С целью изучения особенностей формирования пиноподий у пациенток в различных клинических ситуациях был проведен ряд исследований. Выяснилось, что в естественном цикле образование пиноподий начинается с 5-го дня после овуляции и достигает максимума на 7-й день, после чего пиноподии исчезают в течение 2 суток. При овариальной стимуляции образование пиноподий начинается на 4-й день после назначения ХГЧ и достигает пика на 6-е сутки, после чего количество пиноподий резко уменьшается. В циклах заместительной гормональной терапии (ЗГТ), применяемой в программах переноса замороженных эмбрионов и в программах донации ооцитов, пиноподии появляются на 7-й день после назначения прогестерона, их количество резко возрастает на 8-й день, а затем так же резко снижается. Эти данные говорят о возможном смещении «окна имплантации» в циклах с овариальной стимуляцией, что может объяснять уменьшение вероятности имплантации в таких циклах по сравнению с естественными или циклами с применением 3ГТ [36]. Однако следует отметить, что попытки внедрить исследование пиноподий в клиническую практику не во всех случаях были удачными, и ряд исследователей отмечает, что образование пиноподий не может быть точным маркером «окна имплантации» [37].

Другим маркером имплантации считается появление на эндометрии белков клеточной адгезии, в частности интегринов, которые способствуют прикреплению бластоцисты к поверхности эндометрия [38]. Показано, что экспрессия интегрина avß3 начиналась в естественном цикле на 7-й день после пика ЛГ, тогда как в циклах с применением ова- риальной стимуляции экспрессия этого фактора адгезии начинается с 5-го дня после назначения ХГЧ, что может говорить о смещении «окна имплантации» при стимуляции яичников [39]. Однако несмотря на сообщения об успешном определении интегринов как маркеров имплантации у человека, не все исследователи сообщили о клинической применимости данных методов [40].

\section{МОРФОЛОГИЧЕСКАЯ ХАРАКТЕРИСТИКА}

\section{ЭНДОМЕТРИЯ В СТИМУЛИРОВАННЫХ ЦИКЛАХ}

B 1950 г. R. Noyes описал морфологические изменения эндометрия во время естественного МЦ [41], используя морфологическую хронологическую систему датирования эндометрия в соответствии с учетом МЦ. Согласно этой системе, эндометрий характеризуется как «не в фазе» (out of phase), если расходится с днем цикла по критериям Noyes более чем на 2 дня. По данным ряда исследователей, это связано с тем, что для успешной имплантации важна синхронизация между эмбрионом и эндометрием [16, 27], предполагают, что при опережении развития эндометрия более чем на 3 дня беременность не наступает.

В день пункции фолликулов результаты исследования эндометрия свидетельствуют об опережении его созревания в среднем на 5,8 \pm 0,4 дня в группе пациенток с ант-ГнРГ и на 5,9 \pm 0,7 дня в группе с а-ГнРГ, которое сохранялось до 7-го дня после пункции фолликулов [41]. Как сообщают исследователи [12, 42], результаты биопсии эндометрия в раннюю лютеиновую фазу стимулированного цикла зарегистрировали созревание эндометрия или созревание эндометрия «не в фазе».

A. Tavaniotou и соавторы обнаружили опережение развития эндометрия на 1,8 дня в 100\% случаев, а у половины пациенток без поддержки лютеиновой фазы отмечалась задержка развития эндометрия [16]. При этом эндометрий в позднюю лютеиновую фазу (ХГч + 11-13 дней) соответствовал дню цикла по критериям Noyes, что демонстрировало его восстановление. Кроме того, в эндометрии стимулированных циклов происходит асинхронное созревание желез и стромы, при этом строма более чем на 2 дня опережает созревание желез [16]. В то же время изучение состояния эндометрия у пациенток в день получения ооцитов показало, что асинхронное развитие железистого и стромального компонентов не оказывает
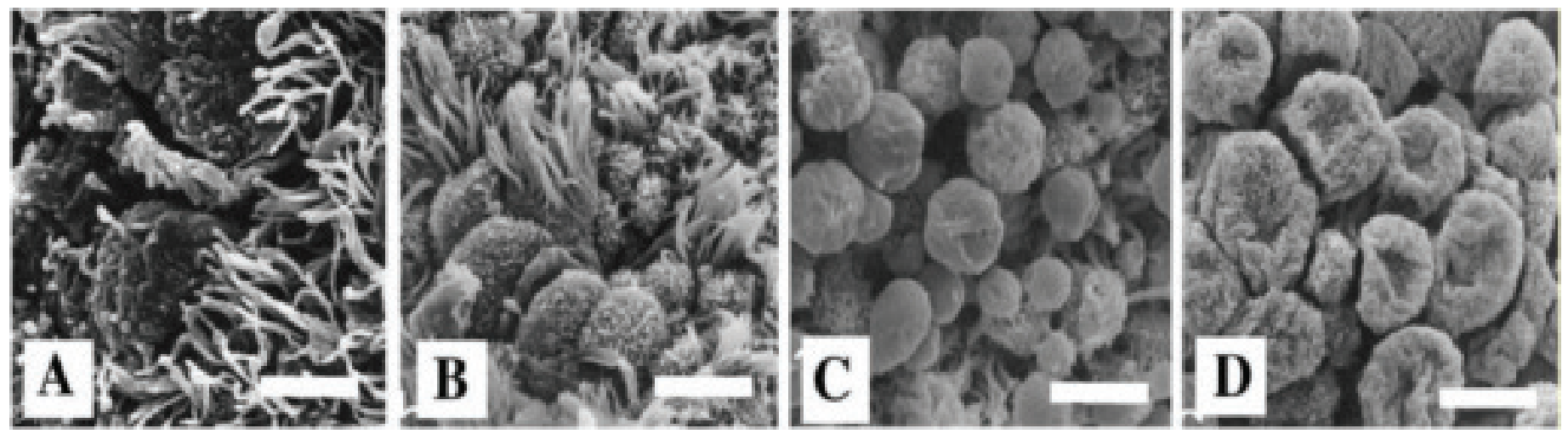

Рисунок 1. Сканирующая электронная микроскопия эндометрия в пролиферативную (A), раннюю (B), среднюю (C) и позднюю лютеиновую фазу (D) нормального МЦ с идентификацией стадии развития пиноподий [56] 
негативного влияния на частоту наступления беременности [43]. Следовательно, эндометрий в стимулированных циклах характеризуется несоответствием дню цикла согласно критериям Noyes и синхронным созреванием желез и стромы.

\section{ЭКСПРЕССИЯ РЕЦЕПТОРОВ Е2 И ПРОГЕСТЕРОНА В НАТУРАЛЬНОМ И СТИМУЛИРОВАННОМ ЦИКЛАХ}

В естественном цикле эстрогеновые рецепторы а и $\beta$ (ЭРа и ЭРß) экспрессируются в эпителиальных и стромальных клетках эндометрия в фазу пролиферации, снижаясь во время фазы секреции $[16,44]$. При этом экспрессия ЭРа максимальна в течение периовуляторного периода [16]. Наивысший уровень экспрессии ЭР $\beta$ обнаруживается в эпителиальных клетках эндометрия в предовуляторном периоде, а также в клетках стромы и эндотелиоцитах в позднюю секреторную фазу. Вышеуказанное исследование демонстрирует, что экспрессия ЭР $\beta$ выражена в значительно меньшей степени, за исключением поздней секреторной фазы МЦ [16]. Исследователи отмечают, что прогестероновые рецепторы (ПР) проиллюстрированы в изоформах А и В. ПР-А и ПР-В экспрессируются в эпителиальных и стромальных клетках в фазу пролиферации, при этом экспрессия обеих форм снижается в эпителиальных клетках во время секреторной фазы цикла, а в строме остается преимущественно ПР-А $[45,46]$.

По данным ряда ученых, уровень экспрессии ЭР выше в пролиферативной фазе, а ПР - в секреторной фазе естественного цикла, достигая максимума во время овуляции [45-47]. Впоследствии экспрессия ЭР и ПР в эпителиальных клетках снижается, в то время как стромальные клетки поддерживают экспрессию ПР и сохраняют чувствительность к прогестерону. Увеличение экспрессии ПР в строме напрямую связано с развитием рецептивности эндометрия [45-47]. Кроме того, ПР-В присутствуют в железах и строме в пролиферативной фазе, резко снижаясь в железах и увеличиваясь в строме в секреторной фазе естественного цикла [45]. Значит, в естественном цикле снижение экспрессии ЭР и ПР в эпителиальных клетках и повышение ПР в строме эндометрия демонстрирует открытие «окна имплантации».

B 2009 г. D. Kyrou и соавторы сообщили, что в фолликулярной фазе стимулированного цикла экспрессия ЭР в железистых и стромальных клетках эндометрия после первоначального увеличения далее значительно не изменяется, при этом экспрессия ПР продолжает увеличиваться [48].

B 2003 г. C. Bourgain и коллеги установили уменьшение экспрессии ПР в железах и строме в периовуляторную и лютеиновую фазы [18]. Другие исследователи выявили снижение ПР в эндометрии после стимуляции суперовуляции в сравнении с натуральным циклом на 4-й день лютеиновой фазы, что не совпадало с обнаруженными морфологическими изменениями [49]. Проведенные в 2001 г. исследования A. Tavaniotou и соавторов показали, что раннее снижение экспрессии ЭР и ПР в железистых клетках в лютеиновую фазу стимулированных циклов приводит к смещению «окна имплантации» вперед [18].
В 2005 г. ученые, занимающиеся данной проблемой, не выявили секреторных изменений в циклах с ант-ГнРГ в поздней фолликулярной фазе при гистологическом исследовании эндометрия в день введения ХГч, при этом экспрессия ПР оказалась значительно выше в железах и строме, в то время как экспрессия ЭР в железах была снижена по сравнению с естественными циклами.

Результаты, касающиеся экспрессии ЭР в эндометрии в стимулированных циклах, значительно варьируют - от полного уменьшения экспрессии до увеличения ее в железах [18]. Значит, несмотря на отсутствие секреторных изменений в эндометрии, экспрессия стероидных рецепторов в стимулированных циклах в день введения триггера соответствовала первым дням лютеиновой фазы в естественных циклах. Поэтому, по мнению D. Kyrou и соавторов, высокий уровень Е2 во время фолликулярной фазы стимулированного цикла вызывает опережение развития эндометрия за счет ранней экспрессии ПР эндометрия [48].

Дальнейшее изучение этого вопроса свидетельствует, что результаты исследований эндометрия в период предполагаемого «окна имплантации» (ЛГ + 7 в естественном цикле и ХГч + 7 в стимулированном цикле) показывают значительное снижение экспрессии ЭРа в стимулированных циклах, при этом отмечена более низкая экспрессия ПР в железах и более высокая в строме [47].

E. Garcia и соавторы сообщают, что снижение экспрессии ПР в железах, но не в строме при яичниковой стимуляции позволяет предположить, что высокий уровень стероидов по-разному воздействует на экспрессию ПР в различных типах клеток [46].

E. Mityurina, S. Perminova информируют об лейкемия-ингибирующем факторе (ЛИФ). ЛИФ - цитокин, который экспрессируется в железистом эпителии и строме эндометрия на протяжении всего МЦ, при этом в пролиферативной фазе его экспрессия минимальна [11]. Авторы [11] указывают, что уровень ЛИФ повышается после овуляции, достигает максимума в среднюю фазу секреции на 19-25-й день и остается относительно высоким почти до конца МЦ [50], снижаясь с началом менструации до базального уровня.

Y. Pan и коллеги сообщают, что экспрессия ЛИФ и его рецепторы на поверхности эндометрия коррелируют с обнаружением пиноподий, причем максимальная их продукция соответствует стадии появления зрелых пиноподий и предполагаемому периоду имплантации бластоцисты [51].

Одним из важных маркеров рецептивности эндометрия являются молекулы клеточной адгезии, среди которых наиболее хорошо изучены интегрины - трансмембранные гликопротеины, состоящие из двух субъединиц (а и $\beta$ ), связанных нековалентными связями. Лигандами интегринов являются фибронектин, витронектин, остеопонтин и коллаген 4-го типа. Авторы обзорной работы отмечают, что рецептор витронектина выявляется в период формирования «окна имплантации», при этом его экспрессия в естественном цикле тесно связана с уменьшением числа ЭР и ПР в эпителии [11]. Секреция лиганда остеопонтина, который предположительно участвует в процессе имплантации эмбриона в эндометрии, находится в прямой зависимости от уровня прогестерона. 
A. Acosta и соавторы сообщают, что интегрины в эндометрии секретируются постоянно, но в то же время экспрессия некоторых из них варьирует в зависимости от фазы МЦ. Исследователи указывают, что экспрессия интегрина av $\beta 3$ у фертильных женщин начинается через 48 часов после появления пиноподий и достигает максимума с 22-го по 28-й день естественного цикла. По данным ряда исследователей, в стимулированных циклах (день ХГч + 7) происходит снижение экспрессии интегринов в железистом эпителии, что, возможно, является одной из причин отсутствия имплантации.

Хорошо известно, что сама по себе овариальная стимуляция часто сопровождается тонким эндометрием. Классическим примером является применение кломифенцитрата (клостильбегита), при использовании которого частота овуляции составляет 50-70\%, а частота наступления беременности - только 5-10\% [4]. Основным фактором, определяющим такую диспропорцию, считается антиэстрогенное действие кломифенцитрата на рецепторы эстрогенов в эндометрии [5]. В последние годы появился ряд исследований, которые показали, что применение кломифенцитрата может вызывать изменения в рецептивности эндометрия. Так, показано, что при применении кломифенцитрата в клетках эпителия эндометрия в середине лютеиновой фазы цикла чаще наблюдаются рецепторы к прогестерону, что сопровождается значительно меньшей экспрессией интегрина $\beta 3$ по сравнению с естественным циклом у тех же пациенток [6].

Вместе с тем роль интегринов в рецептивности эндометрия остается дискуссионной.

\section{МОЛЕКУЛЯРНО-ГЕНЕТИЧЕСКИЕ ИНДУКТОРЫ \\ ЭНДОМЕТРИЯ В СТИМУЛИРОВАННЫХ ЦИКЛАХ}

В последние годы большой интерес вызывает изучение экспрессии генов в различных тканях в норме и при патологии, а также сравнение различий в функции этих генов (геномный подход или геномика). В ходе международного проекта «Геном» было идентифицировано около 30 тысяч генов человека. Тем не менее, в ходе внутриутробного и постнатального развития экспрессируется только 6 тысяч генов. Остальные 24 тысячи представляют собой своеобразный генетический резерв, который может быть реализован при изменении условий существования организма во внутриутробный период и после рождения.

Так, в течение последних 10 лет был проведен ряд исследований, которые показали, что в период имплантации, в середине лютеиновой фазы цикла отмечается повышенная экспрессия определенных генов, функция которых связана с метаболизмом клеток, железистой секрецией, дифференцировкой клеток, межклеточной коммуникацией, врожденным иммунным ответом, ответом на стресс, механизмами заживления ран, механизмами адгезии и регуляцией протеолиза [7]. На основании этих данных было предложено исследование, позволяющее оценить рецептивность эндометрия у конкретной пациентки с помощью изучения экспрессии генов в предполагаемый период имплантации. С этой целью необходимо получить образец эндометрия в естественном цикле на 7-й день после пика ЛГ или в цикле с применением 3ГТ на 5-й день назначения прогестерона. Материал биопсии изучается на предмет экспрессии 238 генов, выбранных путем тщательного анализа. Данный метод, использующий исследование на основе генетического чипа, получил название Endometrial Receptivity Array (ERA) исследование рецептивности эндометрия, основанное на изучении экспрессии массива генов. Авторы утверждают, что их метод позволяет определить наличие рецептивности в конкретном образце эндометрия со специфичностью 0,88 и чувствительностью 0,99 [8]. Клиническую значимость данного метода должны определить дальнейшие исследования.

Из множества публикаций и ряда фундаментальных исследований [2] известно, что в регулировании рецептивности эндометрия участвует порядка 1399 различных генов, из них экспрессия 342 генов увеличивается или уменьшается более чем в 2 раза в стимулированных циклах. При этом среди генов с более активной экспрессией в эндометрии в стимулированных циклах только 46\% являются общими с естественными циклами [1]. Такое мнение ученых иллюстрирует то, что доза гонадотропинов или длительность стимуляции приводит к активации транскрипции генов, которые не вовлечены в рецептивность в физиологических условиях. С другой стороны, 22\% генов имеют менее активную экспрессию в эндометрии в стимулированных циклах, что демонстрирует частичное подавление экспрессии генов при стимуляции функции яичников.

Был исследован профиль экспрессии генов в эндометрии в натуральном цикле (ЛГ + 8) в сравнении с последующим стимулированным циклом (ХГч + 9) в протоколах с а-ГнРГ и ант-ГнРГ, с поддержкой прогестероном лютеиновой фазы или без нее [52]. Как заключили исследователи, несмотря на то, что стимуляция функции яичников вызывает структурные и функциональные изменения в эндометрии, отличия в экспрессии генов были минимальными. В то же время были выявлены существенные изменения при сравнении циклов с использованием а-ГнРГ и ант-ГнРГ (экспрессия 13 генов значительно отличалась).

В 2007 г. J. Horcajadas и соавторы, напротив, обнаружили более 200 генов в эндометрии в стимулированном цикле $($ Хч +7$)$ в сравнении с предшествующим естественным циклом (ЛГ + 7) у тех же женщин [2], при этом в день ХГч + 2 в стимулированных циклах экспрессия генов в эндометрии была сопоставима с естественными (ЛГ + 2). Структура экспрессии генов в «окно имплантации» в протоколах с ант-ГнРГ аналогична таковой в естественных циклах (изменена экспрессия 50 генов) и отличается от длинных протоколов (изменена экспрессия 85 генов).

В дальнейшем исследователи оценивали профиль экспрессии генов в пререцептивной (ЛГ/ХГч +2$)$ и рецептивной $(Л Г+7 /$ ХГч + 5) фазе естественного и последующего стимулированного цикла [1], показав умеренное изменение рецептивности у $86 \%$ и значительное у $14 \%$ пациентов в стимулированных циклах. Данные результаты позволили сделать заключение о необходимости отмены переноса эмбрионов, их криоконсервации и переносе в последующем цикле [1]. В исследовании [53] продемонстрировано, что экспрессия генов эндометрия различна у пациенток с морфологическим опережением созревания эндометрия в день получения ооцитов более чем на 3 дня и без него. 
Группа исследователей провела оценку экспрессии генов маркеров имплантации в зависимости от уровня сывороточного прогестерона в день введения триггера овуляции [45]. В этом плане в 2011 г. R. Li и соавторы оценивали экспрессию генов маркеров имплантации остеопонтина и сосудистого эндотелиального фактора роста (СЭФР) в биоптатах эндометрия на 6-й день после трансвагинальной пункции яичников в зависимости от уровня прогестерона в день ХГч [54]. Было обнаружено увеличение экспрессии генов остеопонтина и уменьшение экспрессии СЭФР у женщин с высокой концентрацией прогестерона (< 4 нмоль/л) в день ХГч, которое исследователи расценили как причину нарушения рецептивности эндометрия, приводящего к незначительным шансам наступления беременности.

Другие ученые изучали данные функционального геномного анализа экспрессии генов эндометрия в «окно имплантации» в зависимости от уровня циркулирующего прогестерона в день введения триггера овуляции в биоптатах эндометрия, полученных в день ХГч + 7 у здоровых доноров ооцитов, подвергшихся стимуляции суперовуляции рекомбинантным фолликулостимулирующим гормоном в протоколах как с а-ГнРГ, так и с ант-ГнРГ [55]. В данной работе исследователи обнаружили значительное изменение экспрессии 140 генов (64 - более активная экспрессия, 76 - менее активная), ответственных за клеточную адгезию, процессы развития иммунной системы и других, необходимых для нормального функционирования эндометрия, в группе пациенток с уровнем прогестерона в день триггера > 1,5 нг/мл по сравнению с контролем независимо от примененного аналога ГнРГ. В своем заключении ученые проинформировали, что повышенный уровень сывороточного прогестерона в день назначения ХГч вызывает огромные изменения в профиле экспрессии генов эндометрия, ответственных за рецептивность.

В 2011 г. I. Van Vaerenbergh и соавторы впервые зарегистрировали существенные различия в профиле экспрессии генов эндометрия в зависимости от уровня прогестерона в назначении ХГч [9]. Исследователи оценивали экспрессию генов эндометрия в день трансвагинальной пункции в циклах с ант-ГнРГ у 14 женщин, разделенных на 3 группы в зависимости от концентрации сывороточного прогестерона в день назначения ХГч: $\leq 0,9$ нг/мл (группа A), 1-1,5 нг/мл (группа В) и > 1,5 нг/мл (группа С). Были обнаружены незначительные отличия в экспрессии генов эндометрия между группами A и B (5 - активная экспрессия и
23 - менее активная в группе В) и выявлены существенные различия между группами В и С (607 - активная экспрессия и 212 - сниженная; $\mathrm{p} \leq 0,045)$.

Представленные результаты, кроме того, иллюстрируют неблагоприятное влияние повышения прогестерона до уровня $\geq 1,5$ нг/мл в день триггера овуляции на рецептивность эндометрия. D. Vitielle и соавторы указывают, что процесс синхронизации между развитием эмбриона и рецептивным эндометрием регулируется молекулярным механизмом, опосредованным гомеобоксными (HOX) генами, которые кодируют индукторы транскрипции [10]. Исследователи отмечают, что экспрессия генов HOXA 10 и HOXA 11 в эндометрии достигает максимума в «окно имплантации» естественного цикла и напрямую зависит от секреции половых стероидов. При анализе экспрессии НОХА 10 в эндометрии в стимулированных циклах в протоколах с ант-ГнРГ и а-ГнРГ изменения экспрессии этого гена обнаружены не были [12]. Вместе с тем известные исследователи B. Rackow и коллеги предполагают, что ант-ГнРГ снижают экспрессию НОХА 10 в строме эндометрия [24]. Поэтому пациенткам с неудачами имплантации в анамнезе перед проведением программы ВРТ ЭКО необходимо провести анализ всех индукторов, влияющих на эндометрий, морфологический пейзаж эндометрия, экспрессию рецепторов E2 и прогестерона, пиноподии, интегрины, молекулярно-генетические аспекты эндометрия в стимулированных циклах.

В заключение целесообразно подчеркнуть, что анализ многочисленных публикаций показывает неблагоприятное влияние стимуляции суперовуляции на рецептивность эндометрия в программах ВРТ. Тем не менее, механизмы этих изменений остаются недостаточно изученными. Кроме того, не определены неинвазивные маркеры для оценки рецептивности эндометрия, в том числе и в стимулированных циклах. Не определен клинически значимый в эндометрии пороговый уровень прогестерона в день введения триггера овуляции и недостаточно исследованы возможные механизмы влияния преждевременного повышения прогестерона на исходы программы ЭКО.

Таким образом, приведенные результаты исхода программы ЭКО демонстрируют необходимость дальнейшего изучения этого вопроса и разработки наиболее оптимальных подходов с целью предотвращения и коррекции негативного влияния стимуляции функции яичников на эндометрий в программах вспомогательных репродуктивных технологий.

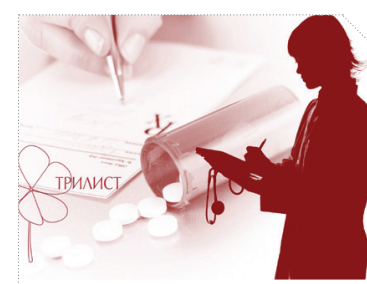

Приведенные результаты исхода программы ЭКО демонстрируют необходимость дальнейшего изучения этого вопроса и разработки наиболее

оптимальных подходов С целью предотвращения и коррекции негативного влияния стимуляции функции

яичников на эндометрий в программах вспомогательных репродуктивных технологий 


\section{ЛИТЕРАTУPA/REFERENCES}

1. Haouzi, D., Assou, S., Mahmoud, K., et al.

"Gene expression profile of human endometrial receptivity:

comparison between natural and stimulated cycles for the same patients." Hum Reprod 24.6 (2009): 1436-45.

2. Horcajadas, J.A., Pellicer, A., Simon, C.

"Wide genomic analysis of human endometrial receptivity: new times, new apportunities." Hum Reprod Update 13.1 (2007): 77-86.

3. Acosta, A.A., Elberger, L., Borghi, M., et al.

"Endometrial dating and determination of the window of implantation in healthy fertile women." Fertil Steril 73.4 (2000): 788-98.

4. Diamond, M.P., Kruger, M., Santoro, N., et al.

"Endometrial shedding effect on conception and live birth in women with polycystic ovary syndrome." Obstet Gynecol 119 (2012): $902-8$

\section{Gonen, Y., Casper, R.F.}

"Sonographic determination of a possible adverse effect of clomiphene citrate on endometrial growth." Hum Reprod 5 (1990): 670-4.

6. Palomino, W.A., Fuentes, A., González, R.R., et al. "Differential expression of endometrial integrins and progesterone receptor during the window of implantation in normo-ovulatory women treated with clomiphene citrate." Ferti Steril 83 (2005): 587-93.

7. Ruiz-Alonso, M., Blesa, D., Simón, C.

"The genomics of the human endometrium." Biochim Biophys Acta 1822 (2012): 1931-42.

8. Garrido-Gómez, T., Ruiz-Alonso, M., Blesa, D., et al. "Profiling the gene signature of endometrial receptivity: clinical results." Fertil Steril 99 (2013): 1078-85.

9. Van Vaerenbergh, I., Fatemi, H.M., Blockeel, C., et al. "Progesterone rise on HCG day in GnRH antagonist/rFSH stimulated cycles affects endometrial gene expression." Reprod Biomed Online 22.3 (2011): 263-71.

10. Vitielle, D., Kodaman, P.H., Taylor, H.S.

"HOX genes in implantation." Semin Reprod Med 25.6 (2007): 431-6.

11. Mityurina, E.V., Perminova, S.G., Demura, T.A., Gallyamova, E.M. "Endometrial receptivity in an in-vitro fertilization program." Obstet Gynecol 2 (2014): 14-20.

12. Taylor, H.S., Daftery, G.S., Selam, B.

"Endometrial HOXA 10 expression after controlled ovarian hyperstimulation with recombinant follicle-stimulating hormone." Fertil Steril 80.2 (2003): 839-43.

13. Edwards, R.G.

"Human implantation: the last barrier in assisted reproduction technologies?" Reprod Biomed Online 13.6 (2006): 887-904.

14. Ferraretti, A., Goossens, V., Bhattacharya, S., et al. Assisted reproductive technology in Europe, 2010: results generated from European registers by ESHRE. Preliminary results. In: European Society of Human Reproduction and Embriology: 29th Annual Meeting. London, United Kingdom, Jul 7-10 2013.

15. Dunson, D.B., Colombo, B., Baird, D.D.

"Changes with age in the level and duration of fertility in the menstrual cycle." Hum Reprod 17 (2002): 1399-403.

16. Tavaniotou, A., Smitz, J., Bourgain, C., Devroey, P. "Ovulation induction disrupts phase function." Ann NY Acad Sci 943 (2001): 55-63.

17. Lessey, B.A.

"The role of the endometrium during embryo implantation." Hum Reprod 15 (2000): 39-50.

18. Bourgain, C., Devroey, P.

"The endometrium in stimulated cycles for IVF." Hum Reprod Update 9.6 (2003): 515-22.

19. Evans, J., Hannan, N.J., Hincks, C., et al.

"Defective soil for a fertile seed? Altered endometrial

development is detrimental to pregnancy success." PLoS One

7.12 (2012): e53098.

20. Noyes, R.W., Hertig, A.T., Rock, J. 262-3.
21. Lessey, B.

"Endometrial integrins and the establishment of uterine

receptivity." Hum Reprod 13.3 (1998): 247-58.

22. Check, J.H., Nowroozi, K., Chase, J., et al.

"Comparison of pregnancy rates following in vitro fertilizationembryo transfer between the donors and recipients in a donor oocyte program."J Assist Reprod Genet 9.3 (1992): 248-50.

23. Shapiro, B.S., Daneshmand, S.T., Garner, F.C., et al. "Evidence of impaired endometrial receptivity after ovarian stimulation for in vitro fertilization: a prospective randomized trial comparing fresh and frozen-thawed embryo transfer in normal responders." Fertil Steril 96.2 (2011): 344-8.

24. Rackow, B.W., Kliman, H.J., Taylor, H.S.

"GnRH antagonists may affect endometrial receptivity." Fertil Steril 89 (2008): 1234-9.

25. Van der Gaast, M.H., Classen-Linke, I., Krusche, C.A., et al. "Impact of ovarian stimulation on mid-luteal endometrial tissu and secretion markers of receptivity." Reprod Biomed Online 17.4 (2008): 553-63.

26. Levi, A.J., Drews, M.R., Bergh, P.A., et al.

"Controlled ovarian hyperstimulation does not adversely affect endometrial receptivity in IVF cycles." Fertil Steril 76.4 (2001): $670-4$.

27. Ubaldi, F., Bourgain, C., Tournaye, H., et al.

"Endometrial evaluation by aspiration biopsy on the day of occyte retrival in the embryo transfer cycle in patients with serum progesterone rise during the follicular phase." Fertil Steril 67.3 (1997): 521-6.

28. Kolibianakis, E., Bourgain, C., Albano, C., et al.

"Effect of ovarian stimulation with recombinant folliclestimulating hormone, gonadotropin releasing hormone antagonist, and human chorionic gonadotropin on endometrial maturation on the day of oocyte pick-up." Fertil Steril 78.5 (2002): 1025-9.

29. Tarlatzis, B.C., Fauser, B.C., Kolibianakis, E.M., et al. "GnRH antagonists in ovarian stimulation for IVF." Hum Reprod Update 12.4 (2006): 333-40.

30. Simon, C., Oberyé, J., Bellver, J., et al.

"Similar endometrial development in oocyte donors treated with either high- or standard dose GnRH antagonist compared to treatment with a GnRH agonist or in natural cycles." Hum Reprod 20.12 (2005): 3318-27.

31. Elnashar, A.M.

"Progesterone rise on day of HCG administration (premature luteinization) in IVF: an overdue update."J Assist Reprod Genet $27.4(2010): 149-55$.

32. Melo, M.A., Meseguer, M., Garrido, N., et al.

"The significance of premature luteinization in an 0ocytedonation programme." Hum Reprod 21.6 (2006): 1503-7.

33. Venetis, C.A., Kolibianakis, E.M., Papanikolaou, E., et al. "Is progesterone elevation on the day of human chorionic gonadotropin administration associated with the probability of pregnancy in in vitro fertilization? A systematic review and meta-analysis." Hum Reprod Update 13.4 (2007): 343-55.

34. Nikas, G.

"Cell-surface morphological events relevant to human implantation." Hum Reprod 14.2 (1999): 7-44.

35. Bentin-Ley, U., Sjögren, A., Nilsson, L., et al.

"Presence of uterine pinopodes at the embryo-endometrial interface during human implantation in vitro." Hum Reprod 14 (1999): $515-20$

36. Nikas, G.

"Pinopodes as markers of endometrial receptivity in clinical practice." Hum Reprod 14.2 (1999): 99-106.

37. Quinn, C., Ryan, E., Claessens, E.A., et al.

"The presence of pinopodes in the human endometrium does not delineate the implantation window." Fertil Steril 87 (2007): 1015-21.

38. Lessey, B., Ilesanmi, A., Sun, J., et al.

"Luminal and glandular endometrial epithelium express integrins differentially throughout the menstrual cycle: implications for implantation, contraception, and infertility." Am J Reprod Immunol 35 (1996): 195-204.
39. Thomas, K., Thomson, A.J., Sephton, V., et al.

"The effect of gonadotrophic stimulation on integrin expression in the endometrium." Hum Reprod 17 (2002): 63-8.

40. Lessey, B.A.

"Assessment of endometrial receptivity." Fertil Steril 96.3 (2011): 522-9.

41. Noyes, R.W., Hertig, A.J., Rock, J.

"Dating the endometrial biopsy." Fertil Steril 1 (1950): 3-25.

42. Saadat, P., Boostanfar, R., Slater, C., et al.

"Accelerated endometrial maturation in the luteal phase of cycles utilizing controlled ovarian hyperstimulation: impact of gonadotropin-releasing hormone agonists versus antagonists." Fertil Steril 82.1 (2004): 167-71.

43. Lass, A., Peat, D., Avery, S., Brinsden, P.

"Histological evaluation of endometrium on the day of oocyte retrieval after gonadotropin - releasing hormone agonistfollicle stimulating hormone ovulation induction in-vitro fertilization." Hum Reprod 13.11 (1998): 3203-5.

44. Papanikolaou, E.G., Bourgain, C., Kolibianakis, E., et al. "Steroid receptor expression in late follicular phase endometrium in GnRH antagonist IVF cycles is already altered, indicating initiation of early luteal phase transformation in the absence of secretory changes." Hum Reprod 20.6 (2005): 1541-7.

45. Gemzell-Danielsson, K., Bygdeman, M.

"Effects of Progestogens on Endometrial Maturation in the Implantation Phase." Ernst Schering Res Found Workshop 52 (2005): 119-38

46. Garcia, E., Bouchard, P., De Brux, J., et al.

"Use of immunocytochemistry of progesterone and estrogen receptors for endometrial dating." J Clin Endocrinol Metab 67.1 (1988): 80-7.

47. Chai, J., Lee, K.F., Ng, E.H., et al.

"Ovarian stimulation modulates steroid receptor expression and spheroid attachment in peri-implantation endometria: studies on natural and stimulated cycles." Fertil Steril 96.3 (2011): 764-8.

48. Kyrou, D., Kolibianakis, E.M., Venetis, C.A., et al. "Steroid receptor expression in human endometrium during the follicular phase of stimulated cycles." Hum Reprod 24.11 (2009): 2931-5.

49. Hadi, F.H., Chantler, E., Anderson, E., et al.

"Ovulation induction and endometrial steroid receptors." Hum Reprod 9.12 (1994): 2405-10.

50. Lessey, B.A., Holoch, K.J., Franasiak, J., et al.

"Prospective assessment of med - secretory endometrial LIF and $\beta 3$ integrin expression as a predictor of pregnancy outcome and endometriosis in unexplained infertility. European Society of Human Reproduction and Embryology: 28th Annual Meeting. Istanbul-Turkey, Jul 1-4, 2012." Hum Reprod 27.1 (2012): 80.

51. Pan, Y.M., Shi, Y.F., Chen, H.Z.

"Expression of estrogen receptor, progesterone receptor and leukemia inhibitory factor on endometrium during different ovarian stimulation protocols in mice." Zhejiang Da Xue Xue Bao Yi Xue Ban 37.3 (2008): 300-3.

52. Mirkin, S., Nikas, G., Hsiu, J.D., et al.

"Gene expression profiles and structural/functional features of the peri-implantation endometrium in natural and gonadotropin-stimulated cycles." J Clin Endocrinol Metab 89.11 (2004): 5742-52.

53. Van Vaerenbergh, I., Van Lommel, L., Ghislain, V., et al. "In GnRH antagonist/rec-FSH stimulated cycles, advanced endometrial maturation on the day of occyte retrieval correlates with altered expression." Hum Reprod 24.5 (2009): 1085-91.

54. Li, R., Oiao, J., Wang, L., et al.

"MicroRNA array and microarray evaluation of endometrial receptivity in patients with high serum progesterone levels on the day of h(G administration." Reprod Biol Endocrinol 9 (2011): 29.

55. Labarta, E., Martínez-Conejero, J.A., Alamá, P., et al. "Endometrial receptivity is affected in women with high circulating progesterone levels at the end of the follicular phase: a functional genomics analysis." Hum Reprod 26.7 (2011): 1813-25. 56. Nikzad, H., et al.

"Expression of galectin-8 on human endometrium: Molecular and cellular aspects." Iran J Reprod Med (2013). 
КЛИНИЧЕСКИЙ ВЗГЛЯД НА ПРОБЛЕМУ НЕЭФФЕКТИВНОСТИ ПОПЫТОК ЭКО ПРИ РЕЦЕПТИВНОСТИ ЭНДОМЕТРИЯ

\section{Обзор литературы}

П.Н. Веропотвелян, к. мед. н., зав. отделением патологии репродуктивной функции человека Межобластного центра медицинской генетики и пренатальной диагностики, г. Кривой Рог

И.С. Цехмистренко, врач акушер-гинеколог Перинатального центра г. Киева

Цель исследования: Провести обзор литературы для изучения механизмов влияния стимуляции суперовуляции на рецептивность эндометрия в программах экстракорпорального оплодотворения (ЭКО), что во многом связано не только с качеством эмбриона, но и с состоянием эндометрия, его зрелостью и готовностью к имплантации.

Учитывая тот факт, что с каждой последующей попыткой вероятность положительного результата ЭКО уменьшается, оптимизация процессов созревания эндометрия, подготовка «окна имплантации», а также поддержка развития беременности на ранних этапах ее развития представляются важнейшими аспектами повышения эффективности вспомогательных репродуктивных технологий (ВРТ). Для успешной имплантации созревание эндометрия, в том числе образование пиноподий и готовность эмбриона, должны быть точно синхронизированы во времени и в пространстве (попадая в «окно имплантации»), что соответствует 19-21 дню менструального цикла.

Материалы и методы: Показаны морфологические характеристики эндометрия в стимулированных циклах. Представлены современные взгляды на рецептивность эндометрия и молекулярные механизмы регуляции имплантации.

Результаты: Описаны особенности экспрессии рецепторов эстрогенов и прогестерона, а также других маркеров рецептивности эндометрия (пиноподии, лейкемияингибирующий фактор, интегрины) в естественных и стимулированных циклах. Пациенткам с неудачами имплантации в анамнезе перед проведением программы ВРТ ЭКО необходимо провести анализ всех индукторов, влияющих на эндометрий, его морфологический пейзаж, экспрессию рецепторов Е2 и прогестерона, пиноподии, интегрины, молекулярно-генетические аспекты эндометрия в стимулированных циклах.

Заключение: Анализ многочисленных публикаций показывает неблагоприятное влияние стимуляции суперовуляции на рецептивность эндометрия в программах ВРТ. Не определены неинвазивные маркеры для оценки рецептивности эндометрия, в том числе и в стимулированных циклах. Не определен клинически значимый в эндометрии пороговый уровень прогестерона в день введения триггера овуляции и недостаточно изучены возможные механизмы влияния преждевременного повышения прогестерона на исходы программы ЭКО. Следовательно, необходимо дальнейшее изучение и разработка наиболее оптимальных подходов с целью предотвращения и коррекции негативного влияния стимуляции функции яичников на эндометрий в программах ВРТ.

Ключевые слова: ЭКО, преждевременная лютеинизация, стимулированный цикл, рецептивность эндометрия, рецепторы эстрадиола, рецепторы прогестерона, интегрины, пиноподии, лейкемия-ингибирующий фактор, экспрессия НОХА.

\section{КЛІНІЧНИЙ ПОГЛЯД НА ПРОБЛЕМУ НЕЕФЕКТИВНОСТІ СПРОБ ЕКЗ ПРИ РЕЦЕПТИВНОСТІ ЕНДОМЕТРІЯ}

\section{Огляд літератури}

П.М. Веропотвелян, к. мед. н., зав. відділенням патології репродуктивної функції людини Міжобласного центру медичної генетики та пренатальної діагностики, м. Кривий Ріг

І.С. Цехмістренко, лікар акушер-гінеколог Перинатального центру м. Києва

Мета дослідження: Провести огляд літератури для вивчення механізмів впливу стимуляції суперовуляції на рецептивність ендометрія в програмах екстракорпорального запліднення (ЕКЗ), що багато в чому пов'язано не тільки з якістю ембріона, а й зі станом ендометрія, його зрілістю і готовністю до імплантації.

3 огляду на той факт, що з кожною наступною спробою ймовірність позитивного результату ЕКЗ зменшується, оптимізація процесів дозрівання ендометрія, підготовка «вікна імплантації», а також підтримка розвитку вагітності на ранніх етапахії розвитку видаються найважливішими аспектами підвищення ефективності допоміжних репродуктивних технологій (ДРТ). Для успішної імплантації дозрівання ендометрія, в тому числі утворення піноподій і готовність ембріона, мають бути точно синхронізовані в часі і в просторі (потрапляючи у «вікно імплантації»), що відповідає 19-21 дню менструального циклу.

Матеріали і методи: Показані морфологічні характеристики ендометрія в стимульованих циклах. Представлені сучасні погляди на рецептивність ендометрія і молекулярні механізми регуляції імплантації.

Результати: Описано особливості експресії рецепторів естрогенів і прогестерону, а також інших маркерів рецептивності ендометрія (піноподії, лейкемія-інгібуючий фактор, інтегрини) в природних і стимульованих циклах. Пацієнткам із невдалими імплантаціями в анамнезі перед проведенням програми ВРТ ЕКО необхідно провести аналіз всіх індукторів, що впливають на ендометрій, його морфологічний пейзаж, експресію рецепторів Е2 і прогестерону, піноподіi, інтегрини, молекулярно-генетичні аспекти ендометрія в стимульованих циклах.

Висновок: Аналіз численних публікацій свідчить про несприятливий вплив стимуляції суперовуляції на рецептивність ендометрія в програмах ВРТ. Не визначені неінвазивні маркери для оцінки рецептивності ендометрія, в тому числі і в стимульованих циклах. Не визначений клінічно значущий в ендометрії пороговий рівень прогестерону в день введення тригера овуляції і недостатньо вивчені можливі механізми впливу передчасного підвищення прогестерону на результати програми ЕКЗ. Отже, необхідне подальше вивчення і розробка найбільш оптимальних підходів з метою запобігання і корекції негативного впливу стимуляції функції яєчників на ендометрій в програмах ВРТ.

Ключові слова: ЕКЗ, передчасна лютеїнізація, стимульований цикл, рецептивність ендометрія, рецептори естрадіолу, рецептори прогестерону, інтегрини, піноподіi, лейкеміяінгібуючий фактор, експресія НОХА.

\section{CLINICAL VIEW OF THE INEFFECTIVENESS OF IVF PROBLEM WITH ENDOMETRIAL RECEPTIVITY}

\section{The literature review}

P.M. Veropotvelyan, PhD, head of the Pathology of Human Reproductive Function Department, Interregional Center of Medical Genetics and Prenatal Diagnostics, Kryvyi Rih

I.S. Tsekhmystrenko, obstetrician-gynecologist at the Perinatal Center of Kyiv

Objective: To review the literature for studying the mechanisms of influence of superovulation on the receptivity of the endometrium in vitro fertilization (IVF), which is largely due not only to the quality of the embryo, but with the condition of the endometrium, its maturity and readiness for implantation.

Given the fact that with each subsequent attempt likelihood of a positive outcome of IVF decreases endometrial maturation process optimization, training of "implantation window", as well as support the development of pregnancy in the early stages of its development represents an important aspect of improving the effectiveness of ART.

Successful implantation endometrium maturation, including the formation and readiness pinopody embryo must be accurately synchronized in time and space (an "implantation window"), which corresponds to 19-21 days menstrual cycle.

Materials and methods: In the course of acquaintance with the publication presents the morphological characteristics of the endometrium in stimulated cycles. Presents current views on endometrial receptivity and the molecular mechanisms regulation of implantation.

Results: The features of the expression of estrogen and progesterone receptors, as well as other markers of endometrial receptivity (pinopodii, leukemia inhibitory factor, integrins) in natural and stimulated cycles. Patients with a history of failure of implantation prior to IVF ART program is necessary to analyze all inducers affecting the endometrium, endometrial morphological landscape, E2 receptor expression and progesterone pinopodii, integrins, molecular and genetic aspects of the endometrium in stimulated cycles.

Conclusion: The analysis of numerous publications showing an adverse effect on superovulation endometrial receptivity in assisted reproduction programs. Undetermined noninvasive markers to assess the receptivity of the endometrium, including in stimulated cycles. Not evaluated linically significant endometrial threshold level of progesterone on the day of ovulation trigger and insufficiently studied the possible mechanisms of the effect of a premature increase of progesterone on the outcomes of IVF. Therefore, further study and development of the best approaches for the prevention and correction of the negative impact of ovarian stimulation function on the endometrium in assisted reproduction programs.

Keywords: IVF, premature luteinization stimulated cycle, endometrial receptivity, receptors estradiol, progesterone receptors, integrins, pinopodii, leukemia inhibitory factor expression of Noja. 